\title{
How oblique trailing edge of a hydrofoil reduces the vortex-induced vibration
}

\author{
A. Zobeiri*, P. Ausoni, F. Avellan, M. Farhat \\ Laboratory for Hydraulic Machines, Ecole Polytechnique Fédérale de Lausanne, 33 bis avenue de Cour, CH-1007 Lausanne, Switzerland
}

\section{A R T I C L E I N F O}

\section{Article history:}

Received 3 November 2010

Received in revised form

27 November 2011

Accepted 1 December 2011

Available online 17 January 2012

\section{Keywords:}

Vortex shedding

Wake

Trailing edge

Flow-induced vibration

Fluid-structure interaction

\begin{abstract}
A B S T R A C T
The effect of hydrofoil trailing edge shape on the wake dynamic and flow induced vibration is investigated at high Reynolds number, $\operatorname{Re}=0.5 \times 10^{6}-2.9 \times 10^{6}$. Two NACA 0009 hydrofoils with blunt and oblique trailing edges are tested. The velocity field is surveyed with the help of Laser Doppler Velocimetry (LDV), and Particle-ImageVelocimetry, (PIV). Proper-Orthogonal-Decomposition (POD) is used to extract coherent structures from PIV data. Besides, flow induced vibration measurements and highspeed visualization are also performed. A significant reduction of vortex induced vibration is obtained with the oblique trailing edge, in accordance with former reports. High speed videos clearly demonstrate that for both tested hydrofoils, the alternate vortices detach from upper and lower corners of the trailing edge. Due to the oblique truncation, the lower detachment location is shifted upstream with respect to the upper one. Therefore, as the upper vortex rolls up, it coincides with the passage of the lower vortex, leading to their collision. This strong interaction leads to a redistribution of the vorticity, which no more concentrates within the core of Karman vortices. The analysis of the phase locked average of velocity profiles reveals that the oblique truncation leads to a thickening of the core of upper and lower vortices as well as a disorganization of the alternate shedding in the near wake, recovers downstream. We strongly believe that the collision between upper and lower vortices and the resulting vorticity redistribution is the main reason of the vibration reduction obtained with oblique trailing edge. This result paves the way for further optimization of the trailing edge shape.
\end{abstract}

(c) 2011 Elsevier Ltd. All rights reserved.

\section{Introduction}

Vortex shedding from hydrofoils trailing edge is an important issue from both scientific and engineering viewpoints. The fluctuating forces associated with such alternate shedding may lead to a significant increase of induced vibrations and risk of premature cracks in a variety of industrial applications, such as hydraulic turbines and pumps as well as marine propellers. The formation process of alternate vortices has been investigated by Roshko (1955), Gerrard (1966), Bearman (1984), Griffin (1995) and Williamson and Roshko (1988) among many others. It is well known that the strong interaction between upper and lower separating shear layers at the hydrofoil trailing edge is the origin of the vortex street formation. When a vortex is initiated in one of these shear layers, it grows and becomes strong enough to draw opposing shear layer across the near wake. The vortex is then shed downstream allowing for a second vortex to form in opposing shear layer. The first theory on stability of a vortex street was proposed by von Karman (Milne-Thomson, 1972), who stated that a

\footnotetext{
* Corresponding author. Tel.: +4121 6933917; fax: +41216933554.

E-mail address: amirreza.zobeiri@gmail.com (A. Zobeiri).
} 
stable vortex shedding is only possible if the vortices are shed alternately and if the ratio between the lateral and longitudinal spacing is equal to 0.28 .

Vortex-induced vibration is noted as important subject in different fields, such as fluid mechanics, structural mechanics, vibrations, computational fluid dynamics and acoustics. Different discussions of these subjects are presented in the reviews of Rockwell (1998), Williamson and Govardhan (2004), Sarpkaya (2004), Bearman (2011) and Assi et al. (2006). Fluid-structure interaction increases vibrations of the structures and may cause structural damage under certain unfavorable conditions. The flow-induced vibration due to vortex shedding is a prevalent fluid-structure interaction problem. For instance, if the shedding frequency coincides with one of the eigenfrequencies of the body, resonance occurs with a significant increase of vibration amplitude. As a result, the structural displacement controls the fluid excitation leading to so-called lock-in phenomenon. It is well known; see for instance Ausoni et al. (2007), that in the case of 2D blunt hydrofoil, the shedding frequency follows a Strouhal law provided that no resonance frequency is excited; i.e., lock-off. Under lock-in condition, the curved vorticity lines turn into straight lines parallel to the trailing edge and the vortex strength is increased (Davies, 1976).

Since vortex-induced vibration can be the reason for damage to different engineering structures, a number of studies attempted to control the wake behind structures, refer to Choi et al. (2008) for an in-depth review. Different methods are proposed to control the wake. For instance, thin splitter plate (Hwang et al., 2003; Ozono, 1999); rotary oscillations of a bluff body (Konstantinidis et al., 2005); acoustic waves (Roussopoulos, 1993); blowing and suction (Park et al., 1994; Cadot and Lebey, 1999); geometry modification in the span-wise direction near the separation point, such as a segmented trailing-edge (Rodriguez, 1991); wavy trailing-edge (Tombazis and Bearman, 1997; Cai et al., 2008); small-size tab, mounted on upper and lower trailing edge (Park et al., 2006); trailing edge shape modification (Donaldson, 1956; Heskestad and Olberts, 1960; Blake, 1986). It is well established that the geometry of the trailing edge has a direct influence on wake structure and vortex-induced vibration level. The vortex shedding from a cambered hydrofoil with beveled trailing edge with different angle at high Reynolds number was studied by Dwayne (2005). He noted that the thicker or blunter trailing edge is producing stronger vortex shedding. Moreover, the boundary layer is separated at the beveled trailing edge and an asymmetric vortex shedding is found. Mosallem (2008) investigated the characteristics of flow past a beveled trailing edges attached to the flat plates with two different angles, $27^{\circ}$ and $60^{\circ}$. The results show an asymmetric wake behind the smaller beveled trailing edge contrary to the greater one that a symmetric wake is observed. The flow past a hydrofoil with blunt and various base cavity shapes at high Reynolds numbers was studied numerically (Do et al., 2010). The base cavity at the trailing edge has effect on the wake structure, decreasing the intensity of the trailing edge pressure fluctuations. As a result, the lift fluctuations reduce. In addition, the study of Lockey et al. (2006) shows that the V-shaped trailing edge reduces the vortex-induced vibration. Donaldson (1956) performed systematic measurements of flow-induced vibration in Francis turbine runners with different trailing edge shapes. He found a significant reduction of vibration with an oblique cut of the blunt trailing edge with an angle of $30^{\circ}$. However, a clear explanation of the physics behind the vibration reduction is still lacking.

The objective of the present study is to investigate the effect of an oblique trailing edge on the wake dynamics to better describe the physical reasons for vibration reduction and allow for further optimization. The case study consists of two similar hydrofoils having blunt and oblique trailing edges and placed in the test section of the EPFL high-speed cavitation tunnel. The survey of the velocity field in the hydrofoil's wake is performed via Laser Doppler Velocimetery (LDV), and Particle-ImageVelocimetry (PIV). The Proper-Orthogonal-Decomposition (POD), is used for PIV data post processing to identify large coherent structures in the wake. In addition, flow-induced vibration and high-speed visualization are also carried out.

\section{Case study and experimental set-up}

Two NACA 0009 hydrofoils with truncated and oblique, $\beta=30^{\circ}$, trailing edges are selected for the present investigation. The hydrofoils are made of stainless steel and they are fixed on one side and free the other side. Both have $100 \mathrm{~mm}$ chord length, $L, 150 \mathrm{~mm}$ span length, $b$, and $10 \mathrm{~mm}$ maximum thickness, $h$, Fig. 1 . Since the state of the boundary layer on the hydrofoil surface is an important parameter for correct comparison between the wakes dynamics of two selected hydrofoils (Ausoni et al., 2007), a special care is taken to ensure a similarity of their surface roughness. The measurements are carried out in the EPFL high-speed cavitation tunnel (Avellan et al., 1987), with a test section of $150 \times 150 \times 750 \mathrm{~mm}{ }^{3}$, maximum inlet velocity, $C_{\text {ref }}$, of $50 \mathrm{~m} / \mathrm{s}$, and maximum static pressure, $P_{\text {inlet }}$, of 16 bars. The free stream turbulence intensity, derived from LDV measurement of the velocity field at test section inlet, was found to be around $1 \%$.

Vortex-induced vibration is monitored on the hydrofoil surface with a Laser vibrometer. The measurement principle of this non-intrusive device is based on the detection of frequency shift between incident and reflected laser beam, Doppler Effect, which is directly related to displacement velocity of the surface. The measurement point is located at mid-span and $10 \%$ of chord length, upstream from the trailing edge. To allow for flow visualization, the wake is made visible by reducing the ambient pressure in the test section so that cavitation can develop within the core of the vortices. According to Ausoni et al. (2007), despite the increase of the vortex shedding frequency, cavitation has almost no effect on the wake structure. A high-speed camera, having an image resolution of $512 \times 256$ pixels at 10000 frames/second frame rate is used to analyze the wake structure.

A survey of the velocity field in the hydrofoil wake is performed with a single-point, two components Laser Doppler velocimeter, LDV, and a 2-D Particle-Image-Velocimeter (PIV). For both methods, hollow glass spheres of $10 \mu \mathrm{m}$ diameter, 

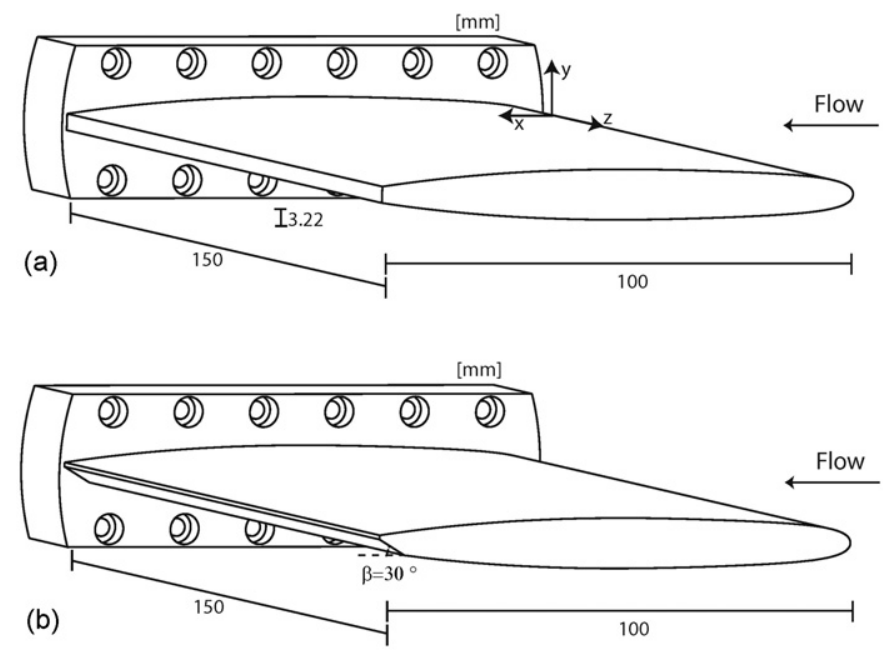

Fig. 1. NACA 0009 hydrofoils (a) truncated trailing edge and (b) oblique trailing edge.

are used as seeding particles. For LDV, the light source is a $10 \mathrm{~W}$ Argon-ion laser, which provides two pairs of green and blue beams. An optical probe, having $250 \mathrm{~mm}$ focal length, is used to focus laser beams in an ellipsoidal measurement volume, which is $75 \mathrm{~mm}$ long and $1.3 \mathrm{~mm}$ wide. Due to random sampling, which is inherent to LDV, particles with high velocity cross the measurement volume more often than particles with low velocity. This leads to a bias in estimating statistical properties of the velocity. We use the particles transit time as a weighting factor to estimate average and Rms values (Buchhave et al., 1979; Herrin and Dutton, 1993).

Phase-locked average of the velocity field is performed to extract the deterministic and periodic component of the signal from the turbulent noise with a good signal to noise ratio. To this end, an accelerometer is used as reference signal, providing a single pulse at every period of vortex generation. The single pulse at every period is generated using pulse generator. It is connected to the LDV processor synchronization input. The phase-locked average procedure is applied directly by sorting the samples into the corresponding phase based on the arrival time. The phase locked average of the velocity, $\bar{C}$, may be defined as follows:

$$
\bar{C}(t)=\lim _{N \rightarrow \infty}\left(\frac{1}{N} \sum_{i=1}^{N} C(t+i T)\right) \quad 0 \leq t<T
$$

where $t$ denotes the time and $T$ is the period of vortex shedding.

In the particular case of discrete and random LDV data, the vortex shedding period is divided into $N_{s}$ segments. The data are then re-arranged using a new time variable $t^{*}$ defined as $t^{*}=t$ modulo $T$. The phase locked average of the velocity for a given segment, $i$, is computed over the velocity samples contained within the segment as follows:

$$
\bar{C}\left(\frac{i}{N_{s}} T\right)=\frac{1}{\sum_{j=1}^{N} W_{j} \Pi_{j}^{i}} \sum_{j=1}^{N} C_{j} W_{j} \Pi_{j}^{i} \quad i=0, \ldots, N_{s}-1,
$$

where $W_{j}$ denotes the particle transit time (weighting factor) and $C_{j}$ is the measured velocity. $\Pi_{j}^{i}$ is a rectangular function equal to 1 inside the segment $i$ and zero outside

$$
\Pi_{j}^{i}= \begin{cases}1 & \text { if } \frac{i}{N_{s}} T \leq t_{j}^{*}<\frac{i+1}{N_{s}} T, \\ 0 & \text { elsewhere, }\end{cases}
$$

Particle-Image-Velocimetry is performed using hollow glass spheres of $10 \mu \mathrm{m}$ diameter as seeding particles. The laser sheet, of $1 \mathrm{~mm}$ thickness, is provided by two ND-YAG pulsed laser sources of $532 \mathrm{~nm}$ wavelength, issued from the bottom window of the test section, along the hydrofoil symmetry plane. These double pulsed lasers may be repeated at a maximum rate of $10 \mathrm{~Hz}$. The pairs of images, captured with an intensified double frame camera, are cross-correlated to derive instantaneous velocity fields. The interrogation area size is $32 \times 32$ pixels with an overlap of $50 \%$. The picture resolution is $1280 \times 1024$ pixels. A Gaussian window function is used to reduce the cyclic noise from the correlation map.

The Proper-Orthogonal-Decomposition (POD) is used as a post processing technique to identify large coherent structures in the wake (for more details, see Sirovich (1987) and Berkooz (1993)). POD is a powerful energy-based filter, which allows for an extraction of the most energetic modes associated with vortex shedding. It also offers the possibility to compute the phase of randomly acquired velocity fields. POD is based on a linear decomposition of velocity fields with 
respect to orthogonal modes,

$$
u^{n}=\sum_{i=1}^{N} a_{i}^{n} \phi^{i}
$$

where $u^{n}$ is a vector representing the velocity components in the entire measurement area and $n$ is the sample index. Given a set of $N$ velocity fields (snapshots), $u^{n}$, a correlation matrix $C$ is defined as

$$
C=U^{\mathrm{T}} U
$$

where $U$ is a matrix made of velocity components of $N$ snapshots. $N$ real positive Eigen values, $\lambda^{i}$, each associated with an eigenvector, $q^{i}$, are obtained from the Eigen value problem.

$$
C q^{i}=\lambda^{i} q^{i},
$$

The normalized POD modes, $\phi^{i}$, are obtained from

$$
\phi^{i}=\frac{\sum_{n=1}^{N} q_{n}^{i} u^{n}}{\left\|\sum_{n=1}^{N} q_{n}^{i} u^{n}\right\|},
$$

The POD coefficient, $a^{n}$, in Eq. (4) presents the projection of the velocity field onto the POD Eigen mode.

$$
a_{i}^{n}=\left(u^{n}, \phi^{i}\right) \text {, }
$$

Exact reconstruction of the velocity field, Eq. (4), may be obtained through a linear combination of $N$ orthogonal modes. The first mode represents the mean flow. The phase of vortex shedding may be derived from the two following modes. In our specific case study, POD method offers an interesting way to perform phase locked averaging of the velocity fluctuation without the need of external trigger.

\section{Results}

We present in Fig. 2 the vortex shedding frequency, derived from LDV measurements, as a function of upstream velocity for both tested hydrofoils. The shedding frequency increases in a quasi linear way with the flow velocity except between 12 and $14 \mathrm{~m} / \mathrm{s}$ for the truncated trailing edge and from 13 to $15 \mathrm{~m} / \mathrm{s}$ for the oblique one. We have already shown (Ausoni et al., 2007) that within these intervals, a hydro elastic coupling occurs (i.e. lock-in) with the vortex shedding frequency being locked onto the hydrofoil eigenfrequency $(890 \mathrm{~Hz})$. The mode shape involved in this case is the torsional mode (Ausoni et al., 2007). Surprisingly, we also observe a similar lock-in in the case of oblique trailing edge for flow velocities between 7 and $9 \mathrm{~m} / \mathrm{s}$ with a shedding frequency of $445 \mathrm{~Hz}$, which is half of the resonance frequency of the torsional mode.

Amplitude spectra and Rms values of vibration signals are plotted in Figs. 3 and 4 respectively, versus upstream velocity for truncated and oblique trailing edge hydrofoils. Both tested hydrofoils exhibit a significant increase of vibration amplitude under lock-in condition within velocity intervals mentioned above. In this case, the vibration of the truncated trailing edge hydrofoil is found at least $50 \%$ higher than the oblique one. Under lock-off conditions, the logarithmic scale in Fig. 4 reveals less vibration with the oblique trailing edge hydrofoil, in line with former reports (Donaldson, 1956; Heskestad and Olberts, 1960). Nevertheless, higher vibration amplitude for oblique trailing edge hydrofoil is also observed for flow velocities between 7 and $9 \mathrm{~m} / \mathrm{s}$, which correspond to the shedding frequency locked

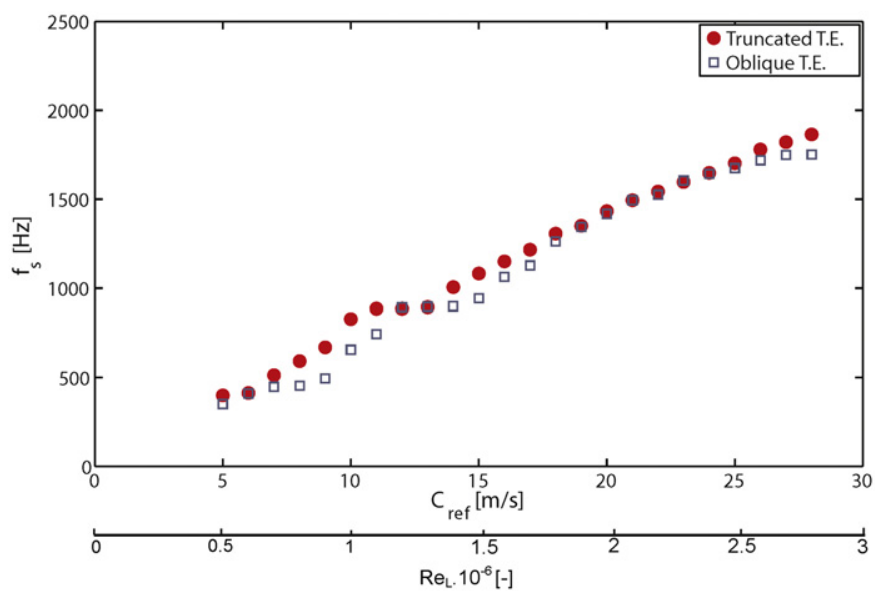

Fig. 2. Shedding frequency versus upstream velocity. 

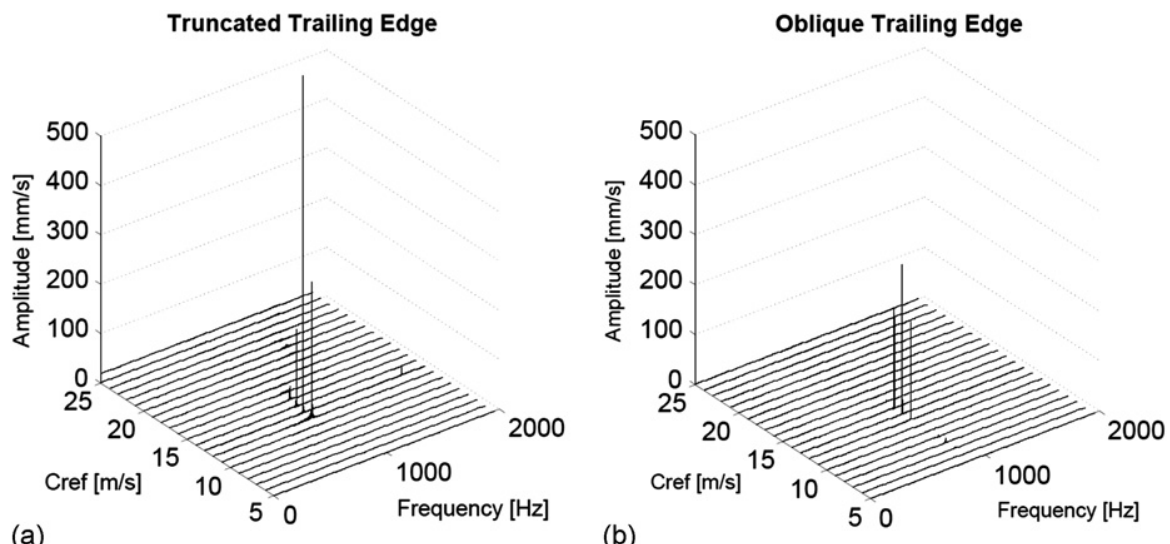

Fig. 3. Amplitude spectra of the vibration signal versus upstream velocity (a) truncated trailing edge and (b) oblique trailing edge.
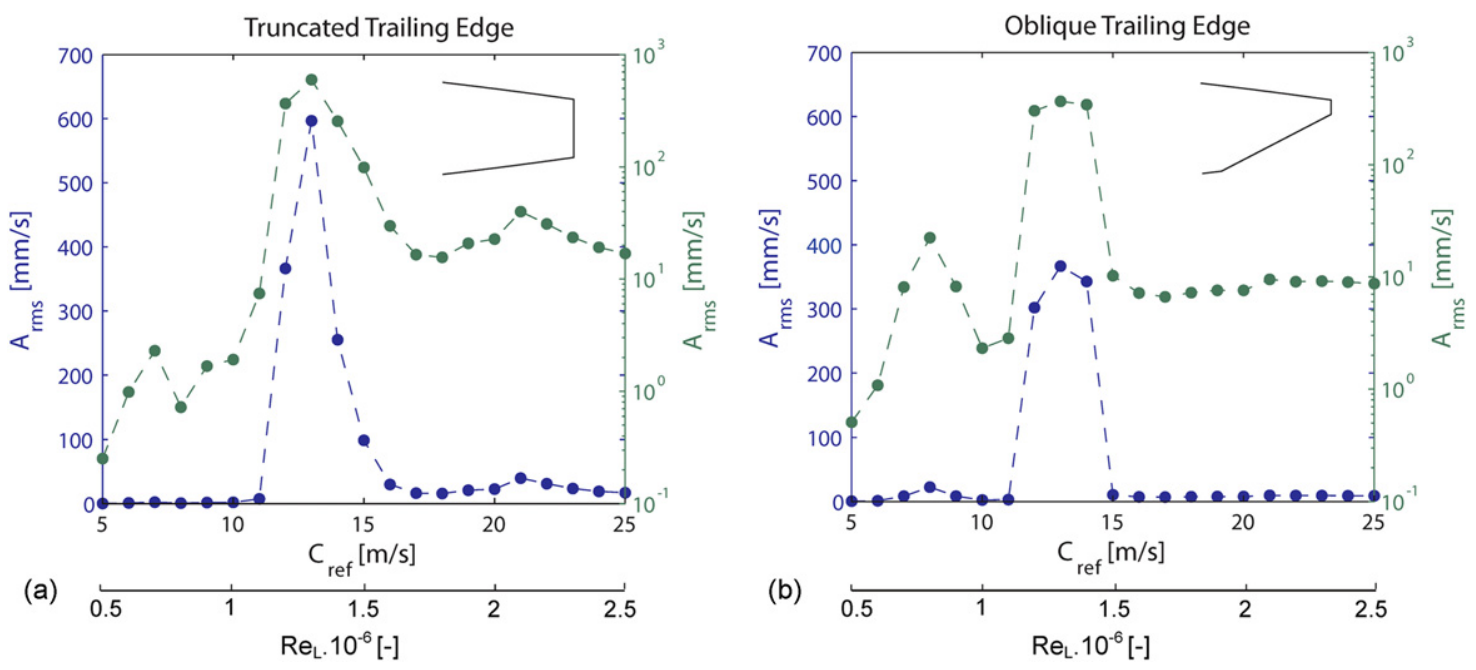

Fig. 4. Rms values of the vibration signal versus upstream velocity, logarithmic and linear scales (a) truncated trailing edge and (b) oblique trailing edge.

onto about half of the resonance frequency, Fig. 2. It should be noticed that this secondary lock-in involves the same torsional mode as with the main lock-in. We believe that this peculiar result is due to the non-symmetric trailing edge, which produces stronger harmonics and thereby more excitation at resonance frequency. Since, in general, hydrofoils are expected to operate outside resonance, we may conclude that oblique truncation is an interesting remedy to reduce the flow induced vibration.

High speed visualization of the cavitating vortices is presented for both tested hydrofoils in Fig. 5 under lock-in condition. We have intentionally selected this particular condition to illustrate the fundamental difference between oblique and truncated trailing edge hydrofoils. In fact, when the vortex shedding frequency is locked on the hydrofoil resonance frequency, the vorticity lines are parallel to the hydrofoil trailing edge (Ausoni, 2009), which makes it easier to observe the wake dynamic and perform measurements of phase locked average of the wake velocity despite the high Reynolds number. The flow visualization shows that for both tested hydrofoils, the boundary layer on pressure and suction sides remains attached to the wall and the alternate vortices clearly detach from upper and lower corners of the trailing edge. However, with the oblique truncation, the location of the lower vortex detachment is obviously shifted upstream with respect to the upper one. As a result, when the upper vortex rolls up, it coincides with the passage of the lower vortex, leading to their collision. This strong interaction leads to a redistribution of the vorticity, which no more concentrates within the core of Karman vortices. A significant part of the vorticity is carried by tiny vortices in random directions. Moreover, vortex collision also leads to a sudden suppression of cavitation in the lower vortex while it persists in the upper one. This is a clear indication of the pressure increase in the core of the lower vortex, which results from the reduction of coherent vorticity. The vortex collision with the resulting vorticity redistribution is thought to be the main reason of vibration reduction with oblique truncation of the trailing edge. 

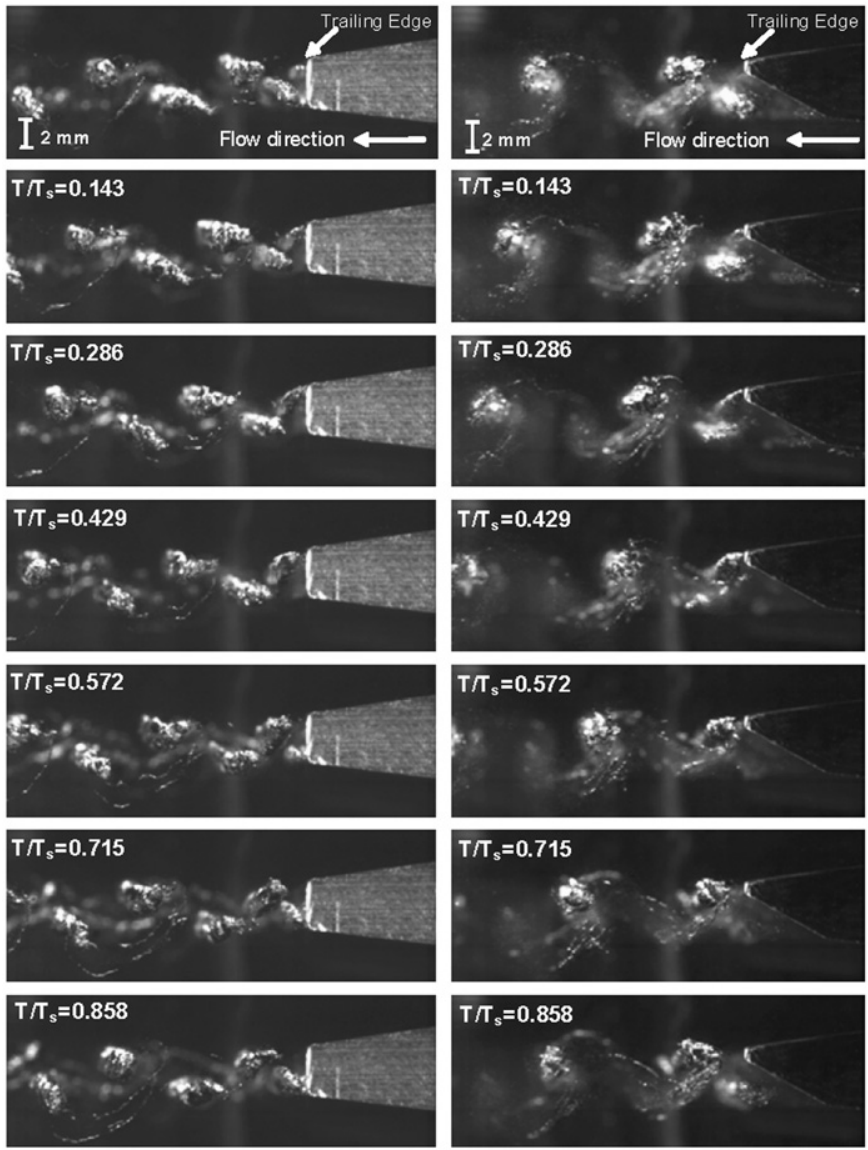

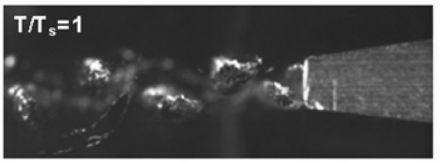

(a)

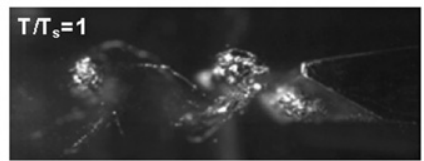

(b)

Fig. 5. High speed visualization of the wake dynamic (side view): (a) truncated trailing edge, $C_{\mathrm{ref}}=12 \mathrm{~m} / \mathrm{s}, \sigma=0.87$ and (b) oblique trailing edge, $C_{\text {ref }}=13 \mathrm{~m} / \mathrm{s}, \sigma=0.6$.

To verify whether the previous analysis based on the visualization of cavitating vortices prevails in cavitation free regime, we have analyzed PIV measurements under lock-in condition for both tested hydrofoils. Phase locked averaging is performed over 1000 snapshots of the velocity field with the help of POD. Random turbulence is filtered by taking into account only the first ten most energetic POD modes, Fig. 6. The snapshots show the vorticity evolution along the wake during one shedding cycle, normalized with chord length and reference velocity. For oblique trailing edge, one may easily observe how upper and lower vortices start building up intensity during their formation stage. As the lower vortex moves forward and collides with the upper one, a significant decrease of its vorticity is observed. The part of the vorticity, which is missing, is redistributed through non-coherent structures and no more appears in the first 10 POD modes. The oblique truncation of the trailing edge is responsible of partial transformation of coherent vorticity into turbulence, which explains the reduction of flow induced vibration. This result, in line with flow visualization of Fig. 5, confirms that cavitation has minor effect on the wake dynamic as already reported by Ausoni et al. (2007).

Moreover, we may also observe from POD plots that the vortex collision occurs during the early stage of the next lower vortex formation. As a result, it slows down the formation process of the later. This is confirmed by high speed visualization, which clearly shows that cavitation is suppresses two times: first during the lower vortex formation and second upon the collision with the upper vortex. In other words, vortex collision weakens not only the colliding vortices but also the next lower vortex.

Further analysis of the wake dynamics is provided by LDV measurements, performed at different sections along the wake with $0.1 \mathrm{~mm}$ vertical resolution. The normalized mean value, $\overline{C_{x}}$, and normalized standard deviation, $C_{x}^{\prime}$, of the stream-wise velocity are illustrated in Fig. 7 for the truncated and oblique trailing edges under lock-in condition. These values are estimated over a minimum of 90000 samples and normalized by the free-stream velocity. Unlike the truncated 

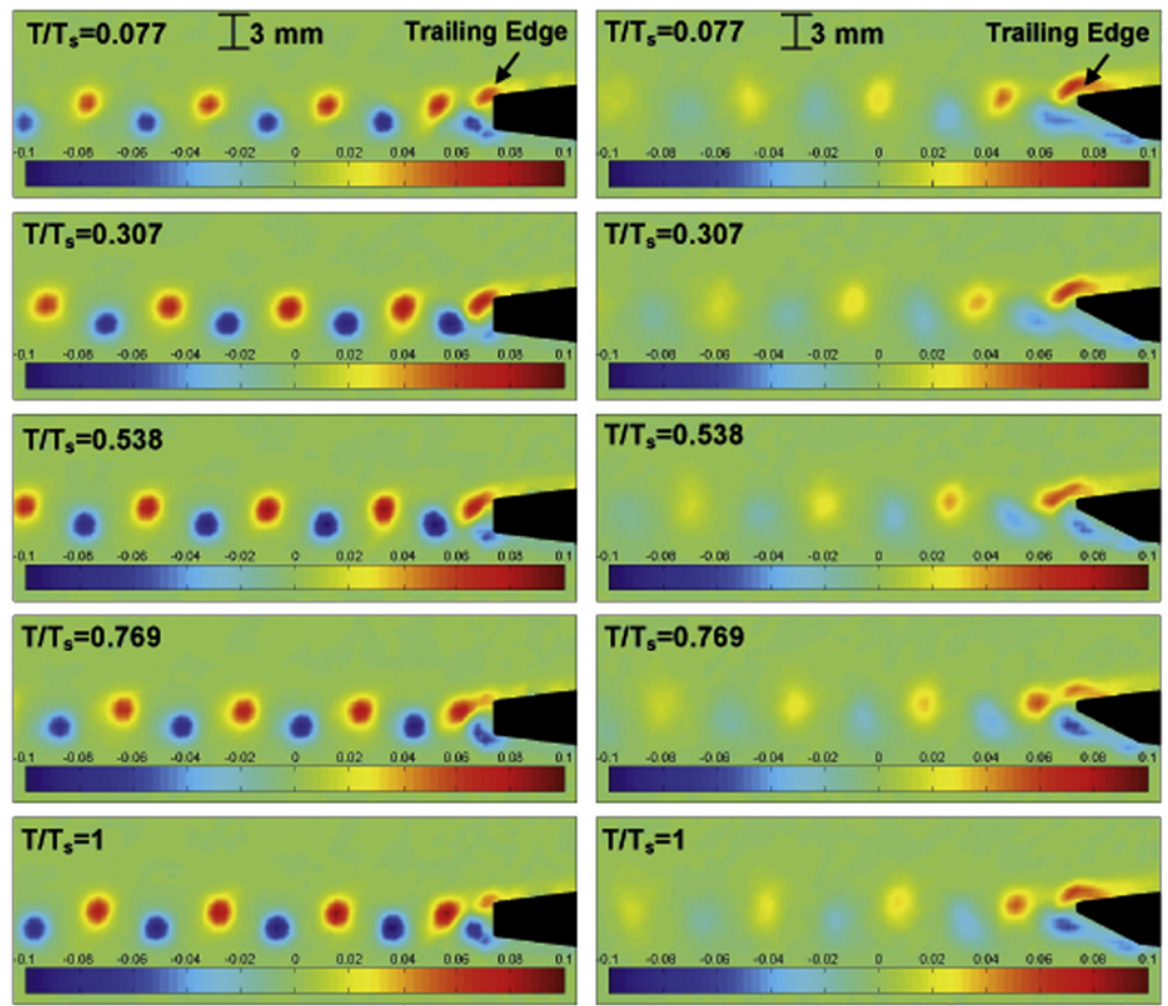

(a)

(b)

Fig. 6. Wake visualization (one shedding period), normalized vorticity (a) truncated trailing edge, $C_{\text {ref }}=12 \mathrm{~m} / \mathrm{s}$ and (b) oblique trailing edge, $C_{\text {ref }}=13 \mathrm{~m} / \mathrm{s}$.

(a)
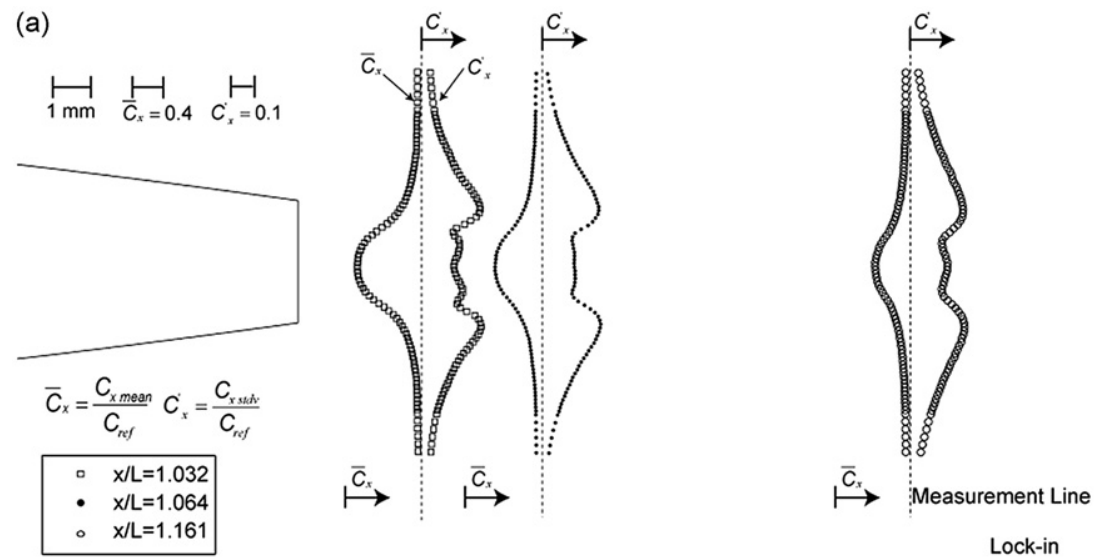

(b)
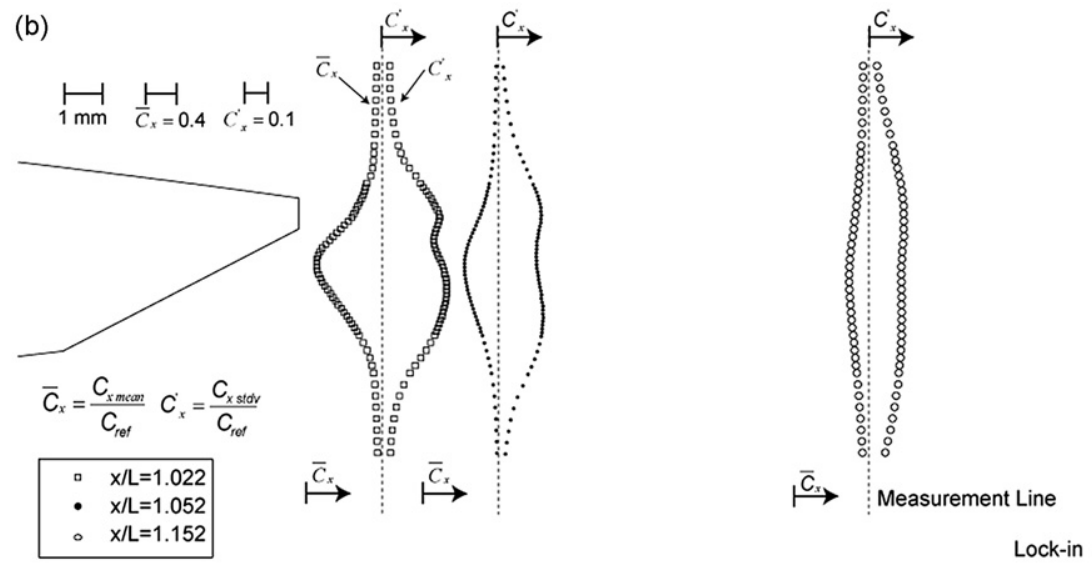

Fig. 7. Velocity measurements at 3 stations under lock-in condition. Normalized mean and standard deviation of stream-wise velocity (a) truncated trailing edge, $C_{\text {ref }}=12 \mathrm{~m} / \mathrm{s}$ and (b) oblique trailing edge, $C_{\mathrm{ref}}=13 \mathrm{~m} / \mathrm{s}$. 
(a)
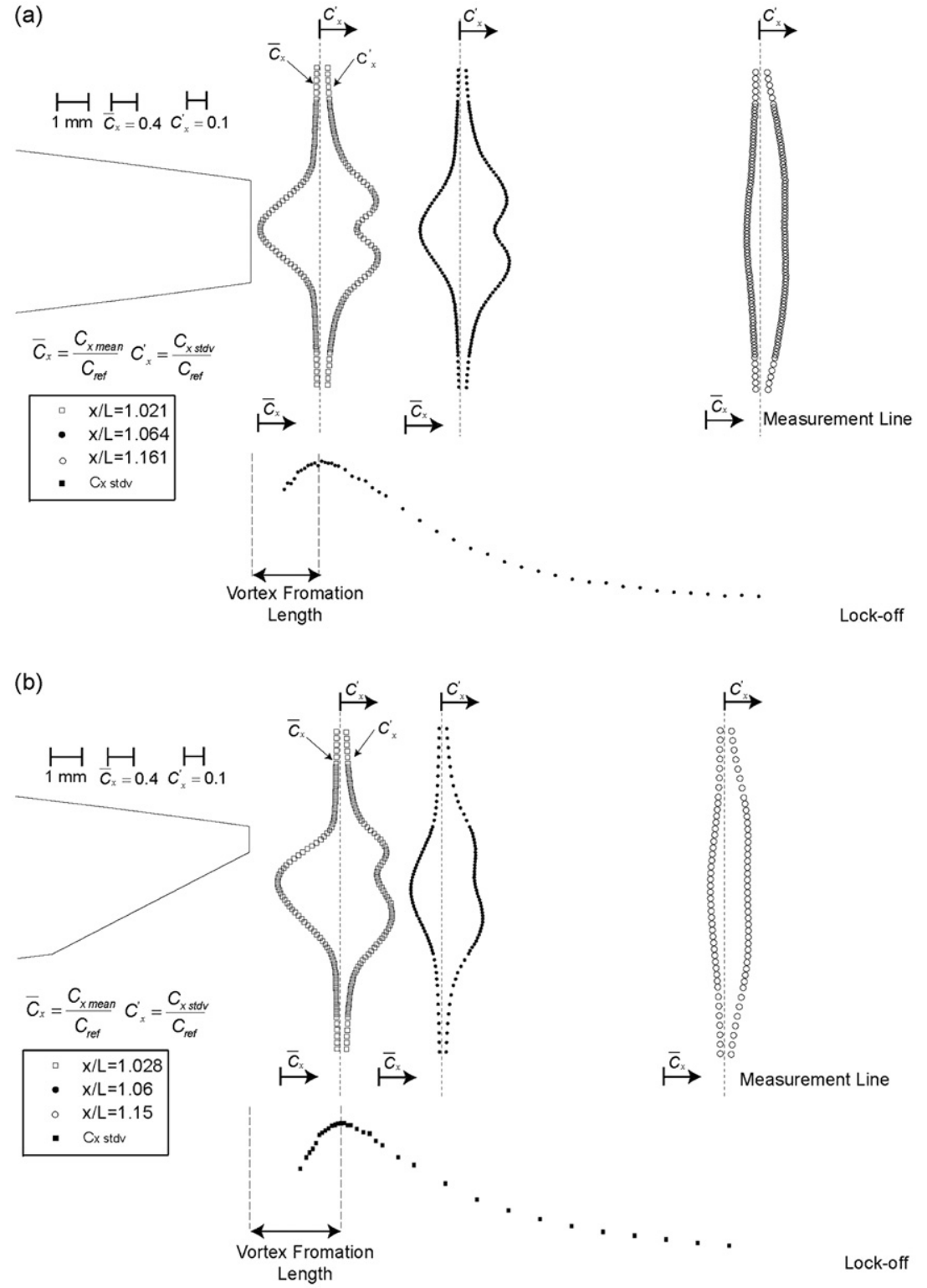

Fig. 8. Velocity measurements at 3 stations under lock-off condition, $C_{\text {ref }}=20 \mathrm{~m} / \mathrm{s}$. Normalized mean and standard deviation of stream-wise velocity (a) truncated trailing edge and (b) oblique trailing edge.

trailing edge, the oblique trailing edge produces an asymmetric thickening of the wake downward. The stream-wise velocity fluctuation exhibits two peaks of different amplitudes, which originate from upper and lower vortex passing. In the far wake, although a decrease of velocity deficit is observed for both hydrofoils, this decrease is more pronounced for the oblique one. The thickening of the lower part of the wake may be explained by an increase of the lower vortex core size, which results from its collision with the upper one, as already suggested by cavitation suppression observed in Fig. 5.

LDV measurements were also carried out under lock-off conditions to confirm the flow analysis presented above. The mean value and standard deviation of the stream-wise velocity at $20 \mathrm{~m} / \mathrm{s}$ upstream velocity are presented in Fig. 8 at 3 different locations of the wake. We have superposed in the same figures the stream-wise velocity fluctuation along the wake center line. These curves are used to localize the end of vortex formation region, which corresponds to the maximum of velocity fluctuation (Griffen, 1995). It should be noticed that the first LDV measurement section corresponds to the end of vortex formation region. This allows for fair comparison of wake dynamic between tested hydrofoils. We have superposed in Fig. 9 the mean value and standard deviation of the stream-wise and transverse velocity at the end of the vortex formation region corresponding to truncated and oblique trailing edges. The asymmetric thickening of the lower part of the wake as well as the reduction of velocity fluctuation is once more highlighted for the oblique truncation. 

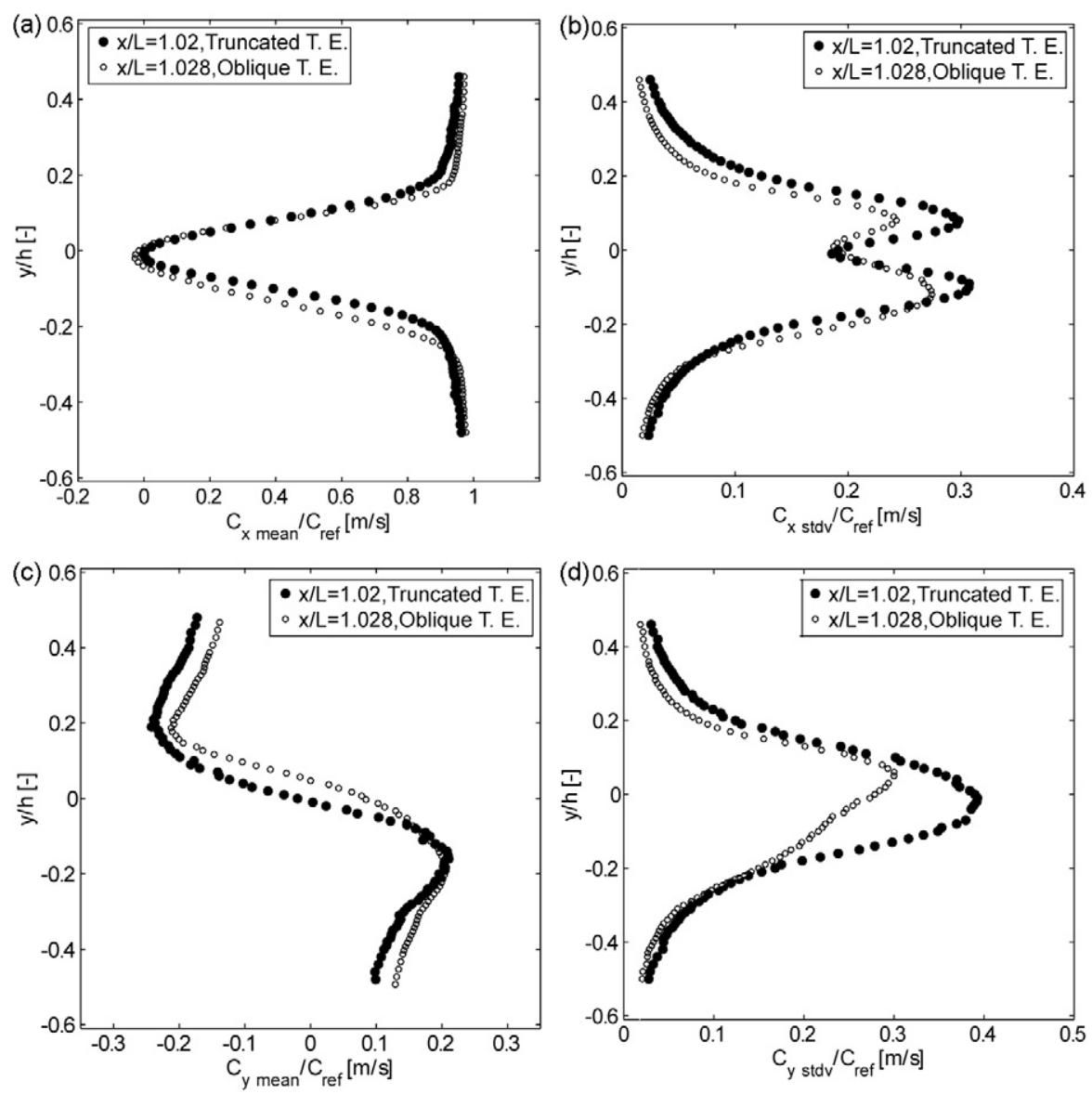

Fig. 9. Velocity measurements across the wake at vortex formation position under lock-off condition, $C_{\text {ref }}=20 \mathrm{~m} / \mathrm{s}$ (a) normalized mean stream-wise, (b) stream-wise velocity fluctuations, (c) normalized mean transverse and (d) transverse velocity fluctuations.
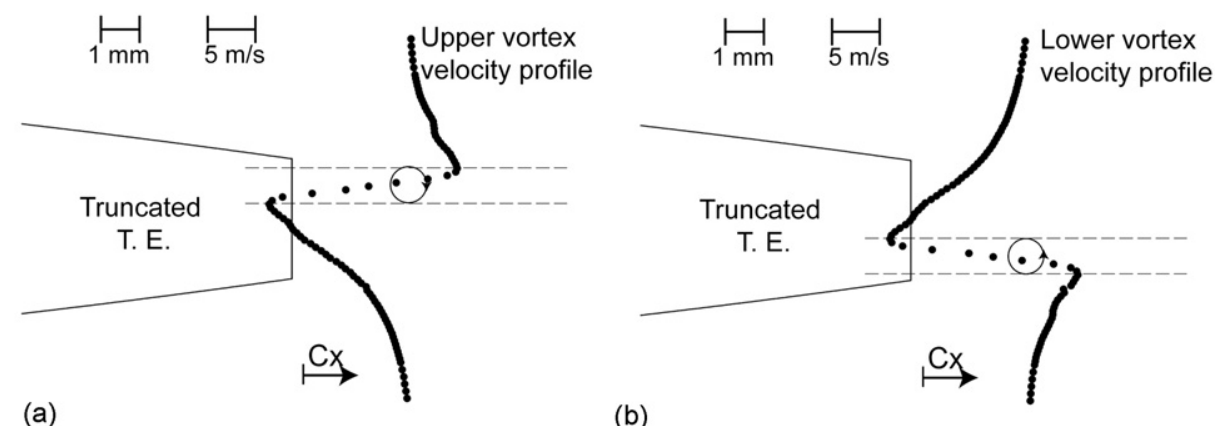

(b)
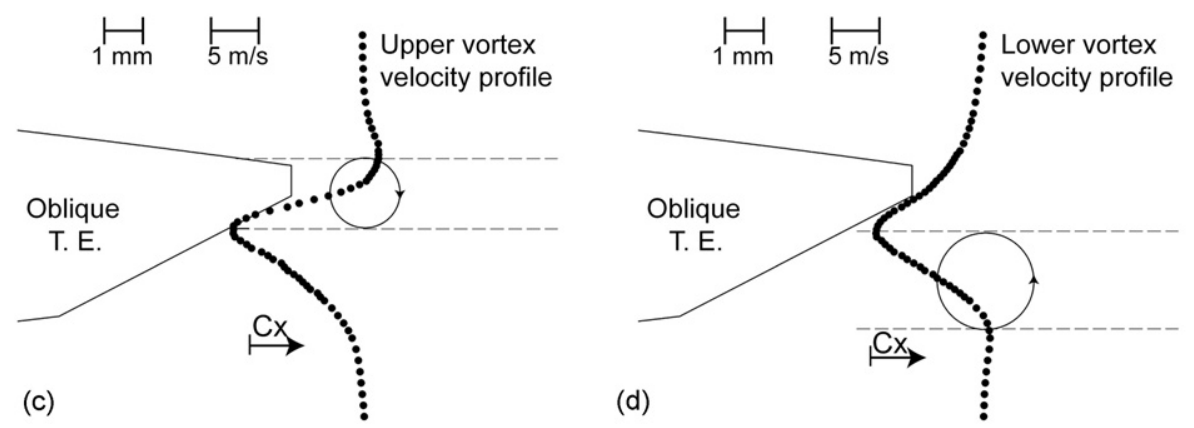

Fig. 10. Stream-wise velocity profile at the passage time of the vortex through the measurement line and vortex core size for truncated and oblique trailing edges, lock-in. 


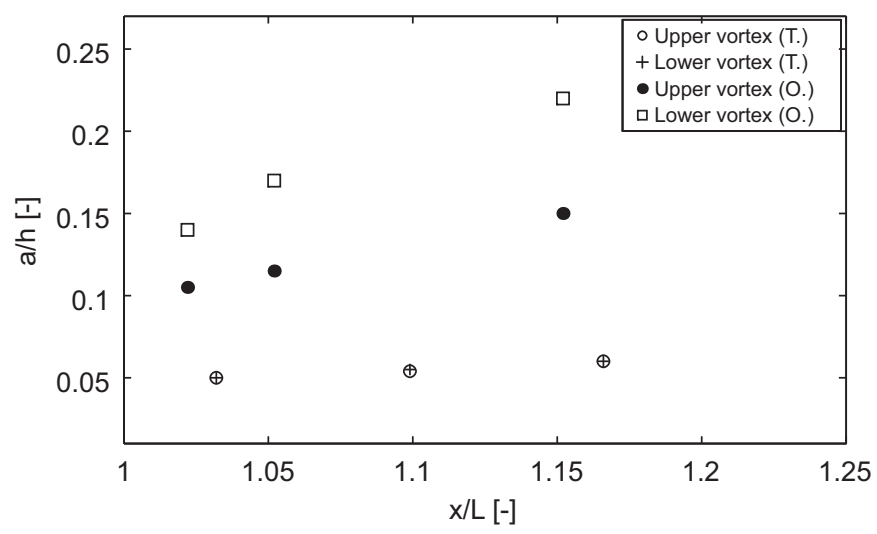

Fig. 11. Vortex core radiuses at different stations downstream from the trailing edge, lock-in.

(a)
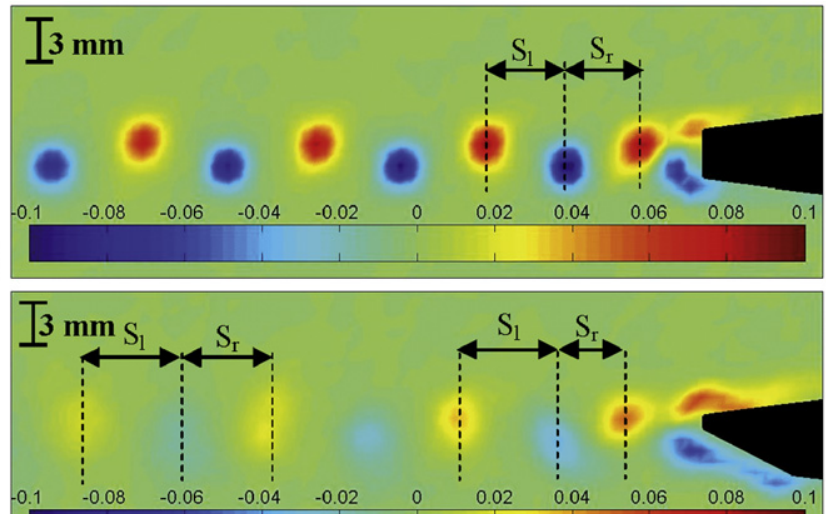

(b)

Fig. 12. Vortex arrangement in the wake of (a) truncated trailing edge and (b) oblique trailing edge, lock-in.

The thickening of the wake and the decay of the velocity deficit in the case of oblique trailing edge are found similar to the lock-in condition. On the contrary, for the blunt hydrofoil, the velocity deficit decreases faster and the wake is thinner under lock-off condition. This may be explained by the strong fluid structure interaction which occurs with the blunt hydrofoil at lock-in. In fact, displacement amplitudes as high as $20 \%$ of the trailing edge thickness were measured. This leads to an increase of the distance between upper and lower vortices and thereby the wake width. This analysis indicates that the vortex collision and the resulting vorticity redistribution, clearly observed under lock-in, prevail also for lock-off condition.

Instantaneous stream-wise velocity profiles at the passage time of the upper and lower vortices through the measurement line may be recovered from LDV measurements through phase locked averaging. To this end, the vibration signal, provided by the laser vibrometer, is used for the phase reference. This procedure is applied only for lock-in condition since it requires parallel and periodic shedding. The result is plotted in Fig. 10. The velocity profiles associated with upper and lower vortex passages are perfectly similar for the blunt trailing edge hydrofoil. This is not the case for oblique one. We have estimated the viscous core of the vortices by simply measuring the distance between the locations of maximum and minimum stream-wise velocities. In the case of a truncated trailing edge, the upper and lower vortices have the same core diameter. For the oblique trailing edge hydrofoil, the viscous core of the lower vortex is larger than the upper one. Both vortices exhibit a larger core than with the truncated trailing edge hydrofoil. This result reveals that upon their collision, both upper and lower vortices see their core size unequally increased, which is an indication of a reduction of their vorticity.

The core diameter of the vortices is estimated at the three measurement sections and its evolution in the wake is presented in Fig. 11. For both tested hydrofoils, the vortex core size increases along the wake. This trend is significantly more pronounced for the oblique trailing edge hydrofoil.

Another consequence of the vortex collision is a disorganization of vortex shedding in the near wake as illustrated in Fig. 12. An alteration of the spatial periodicity in the case of oblique truncation is clearly visible by comparing the distances, $S_{1}$ and $S_{r}$, between the lower vortex and two neighboring upper vortices at the right and left side, respectively. While $S_{1}$ and $S_{r}$ are different in the near wake, they tend to equalize downstream where more organized and alternate shedding is recovered. 


\section{Conclusions}

We have investigated the role of the shape of a hydrofoil trailing edge on vortex shedding and flow induced vibration. Two similar NACA 0009 hydrofoils with truncated and oblique trailing edges are tested in a high speed hydrodynamic tunnel. The main objective is to better explain the physics behind the vibration reduction usually obtained with an oblique truncation of the trailing edge. Experiments are performed for zero incidence angle and high Reynolds numbers, $\mathrm{R}_{\mathrm{eL}}=5 \times 10^{5}-2.9 \times 10^{6}$. LDV and PIV techniques are used for the survey of flow velocity field in the wake. Besides, highspeed visualization and flow induced vibration measurements are also carried out. The main conclusions may be summarized as follows:

- Flow induced vibration is significantly reduced with the oblique trailing edge compared to the truncated one, in line with former reports.

- High-speed visualization of cavitating vortices, in the particular case of lock-in, clearly shows that for both tested hydrofoils, the alternate vortices detach from upper and lower corners of the trailing edge. With the oblique truncation, the lower detachment location is shifted upstream with respect to the upper one. As a result, when the upper vortex rolls up, it coincides with the passage of the lower vortex, leading to their collision.

- PIV measurements, performed in cavitation free and lock-in condition, confirm the vortex collision visually observed on cavitating vortices. Energy based filtering of PIV data, performed with POD technique, shows that the vortex collision results in a redistribution of the vorticity.

- LDV measurements clearly show weaker and thicker vortices with the oblique trailing edge for both lock-in and lock-off conditions. Moreover, the unequal distance between the lower vortex and two neighboring upper vortices in the near wake, introduced by the oblique truncation of the trailing edge, is found to vanish in the far wake, where more organized shedding is recovered, as observed for the blunt trailing edge.

These results let us believe that the collision between upper and lower vortices and the resulting vorticity redistribution is the main reason of the vibration reduction obtained with oblique trailing edge. This result paves the way for more optimization of hydrofoils shape for further reduction of flow induced vibration.

\section{Acknowledgment}

The present investigation was carried out in a partnership with ALSTOM Hydro, ANDRITZ Hydro, VOITH Hydro and UPC-CDIF. The authors would like to thank the Swiss Federal Commission for the Technology and Innovation (CTI), Swisselectric Research, and Swiss Competence Center of Energy and Mobility (CCEM) for their financial support.

\section{References}

Assi, G.R.S., Meneghini, J.R., Aranha, J.A.P., Bearman, P.W., Casaprima, E., 2006. Experimental investigation of flow-induced vibration interference between two circular cylinder. Journal of Fluids and Structures 22, 819-827.

Ausoni, P., Farhat, M., Escaler, X., Egusquiza, E., Avellan, F., 2007. Cavitation influence on Kármán vortex shedding and induced hydrofoil vibrations Journal of Fluids Engineering 129, 966-973.

Ausoni, P., 2009. Turbulent Vortex Shedding from a Blunt Trailing Edge Hydrofoil. PhD thesis, EPFL, No. 4475

Avellan, F., Henry, P., Ryhming, I., 1987. A new high speed cavitation tunnel. American Society of Mechanical Engineers, Fluids Engineering Division 57, 49-60.

Bearman, P.W., 1984. Vortex shedding from oscillating bluff bodies. Annual Review of Fluid Mechanics 16, 195-222.

Bearman, P.W., 2011. Circular cylinder wakes and vortex-induced vibration. Journal of Fluids and Structures 27, 648-658.

Berkooz, G., 1993. The proper orthogonal decomposition in the analysis of turbulent flows. Annual Review of Fluid Mechanics 25, 539-575.

Blake, W.K., 1986. In: Mechanism of Flow Induced Sound and Vibration Academic Press INC, Orlando, United States of America, pp. 757.

Buchhave, P., George Jr., W.L., Lumley, J.L., 1979. The measurement of turbulence with the laser-Doppler anemometer. Annual Review of Fluid Mechanics $11,443-503$.

Cadot, O., Lebey, M., 1999. Shear instability inhibition in a cylinder wake by local injection of a viscoelastic fluid. Physics of Fluids 11, 494-496.

Cai, J., Chng, T.L., Tsai, H.M., 2008. On vertical flows shedding from a bluff body with a wavy trailing edge. Physics of Fluids 20, 064102-064113.

Choi, H., Jeon, W.P., Kim, J., 2008. Control of flow over a bluff body. Annual Review of Fluid Mechanics 40, 113-139.

Davies, M.E., 1976. Comparison of the wake structure of a stationary and oscillating bluff body, using a conditional averaging technique. Journal of Fluid Mechanics 75, 209-231.

Do, T., Chen, L., Tu, J., 2010. Numerical study of turbulent trailing-edge flows with base cavity effects using URANS. Journal of Fluids and Structures 26 $1155-1173$

Donaldson, R.M., 1956. Hydraulic turbine runner vibration. Journal of Engineering for Power 78, 1141-1147.

Dwayne, A., 2005. Vortex shedding from a hydrofoil at high Reynolds number. Journal of Fluid Mechanics 531, 293-324.

Gerrard, J.H., 1966. The mechanics of the formation region of vortices behind bluff bodies. Journal of Fluid Mechanics 25, 410-413.

Griffen, O.M., 1995. A note on bluff body vortex formation. Journal of Fluid Mechanics 284, 217-224.

Herrin, J.L., Dutton, J.C., 1993. An investigation of LDV velocity bias correction techniques for high-speed separated flow. Experiments in Fluids 14 354-363.

Heskestad, F., Olberts, D.R., 1960. Influence of trailing edge geometry on hydraulic turbine blade vibration. Journal of Engineering for Power 82, 103-110. Hwang, J.Y., Yang, K.S., Sun, S.H., 2003. Reduction of flow-induced forces on a circular cylinder using a detached splitter plate. Physics of Fluids 15 2433-2436.

Konstantinidis, E., Balabani, S., Yianneskis, M., 2005. The timing of vortex shedding in a cylinder wake imposed by periodic inflow perturbations. Journal of Fluid Mechanics 543, 45-55. 
Lockey, K.J., Keller, M., Sick, M., Staehle, M.H., Gehrer, A., 2006. Flow-induced vibrations at stay vanes: experience on site and CFD simulations. International Journal on Hydropower and Dams 5, 102-106.

Milne-Thomson, L.M., 1972. Theoretical Hydrodynamics. Macmillan, London, pp. 375.

Mosallem, M., 2008. Numerical and experimental investigation of beveled trailing edge flow fields. Journal of Hydrodynamics $20,273-279$.

Ozono, S., 1999. Flow control of vortex shedding by a short splitter plate asymmetrically arranged downstream of a cylinder. Physics of Fluids 11 , 2928-2934.

Park, D.S., Ladd, D.M., Hendricks, E.W., 1994. Feedback control of von Karman vortex shedding behind a circular cylinder at low Reynolds numbers. Physics of Fluids 6, 2390-2405

Park, H., Lee, D., Jeon, W.P., Hahn, S., Kim, J., 2006. Drag reduction in flow over a two-dimensional bluff body with a blunt trailing edge using a new passive device. Journal of Fluid Mechanics 563, 389-414.

Rockwell, D., 1998. Vortex-body interactions. Annual Review of Fluid Mechanics 30, 199-229.

Rodriguez, O., 1991. Base drag reduction by the control of three-dimensional unsteady vortical structures. Journal of Experiments in Fluids $11,218-226$.

Roshko, A., 1955. On the wake and drag of bluff bodies. National Advisory Committee for Aeronautics 22, 1-39.

Roussopoulos, K., 1993. Feedback control of vortex shedding at low Reynolds numbers. Journal of Fluid Mechanics 248, $267-296$.

Sarpkaya, T., 2004. A critical review of the intrinsic nature of vortex-induced vibrations. Journal of Fluids and Structures 19 , $389-447$.

Sirovich, L., 1987. Turbulence and the dynamics of coherent structures. Journal of Fluid Mechanics 45, 561-571.

Tombazis, N., Bearman, P.W., 1997. A study of three-dimensional aspects of vortex shedding from a bluff body with a mild geometric disturbance. Journal of Fluid Mechanics 330, 85-112.

Williamson, C.H.K., Roshko, A., 1988. Vortex formation in the wake of an oscillating cylinder. Journal of Fluids and Structures 2, $355-381$.

Williamson, C.H.K., Govardhan, R., 2004. Vortex-induced vibrations. Annual Review of Fluid Mechanics 36, $413-455$. 